\title{
hétérogénéités dans les massifs rocheux et stabilité des tunnels
}

\author{
par \\ Dejean M. \\ Docteur Ingénieur, Ingénieur Civil des Mines \\ Centre d'Études et de Recherches \\ des Charbonnages de France \\ Laboratoire de Mécanique des Terrains \\ École des Mines de Nancy \\ et \\ Duffaut P.* \\ Ingénieur Civil des Mines \\ Division Techniques d'exécution \\ Électricité de France
}

\section{Introduction}

II est d'usage de présenter le massif rocheux naturel comme un milieu hétérogène, anisotrope et discontinu, et de considérer ces trois caractères comme autant de défauts susceptibles d'en compromettre la stabilité. II s'agit assurément de caractères qui rendent le comportement du massif rocheux plus complexe que celui du milieu idéal auquel s'appliquent d'abord les théories des mécaniciens, élasticité (résistance des matériaux) plasticité, etc. Mais dans certains cas au moins, ce comportement complexe peut se révéler plus favorable que celui du matériau idéal, et ceci est particulièrement fréquent dans le cas de cavités isolées.

A vrai dire, le matériau idéal continu, homogène et isotrope n'existe nulle part dans la nature et il n'est pas non plus facile de le fabriquer. Tous les matériaux de construction, qu'ils soient naturels ou artificiels, présentent au moins à certaines échelles des discontinuités, des hétérogénéités et/ou des anisotropies. Le constructeur a dâ s'accomoder de ces caractères et dans bien des cas il a su en tirer avantage.

Ainsi le bois a-t-il pu être employé à la fois comme poteau, parce qu'il est rigide dans la direction des fibres, et comme cale ou coin, parce qu'il peut s'écraser considérablement dans la direction perpendiculaire. Ses fibres lui permettent en outre de travailler à la flexion. L'acier et le béton sont au contraire employés à une échelle où leurs hétérogénéités sont négligeables, mais ils ont été associés dans le béton armé afin d'obtenir un comportement satisfaisant en flexion. De même, l'industrie moderne sait associer des matériaux élémentaires très différents pour former volonté des matériaux à propriétés spécifiées, les composites. Tous sont fortement anisotropes, et ces caractères sont justement recherchés par leurs utilisateurs.

Les discontinuités n'apparaissent généralement qu'à l'échelle au-dessus, lorsque des éléments sont assemblés pour former une construction, par exemple dans la construction en pierres sèches. L'influence néfaste des discontinuités est alors limitée, soit par simple orientation des joints perpendiculaires à la compression, soit par imbrication des surfaces en contact, soit par collage, soit par divers autres procédés de fixation (boulons, tirants, etc.).

- Monsieur Duffaut est actuellement Chef du Génie Géologique au Bureau de Recherches Géologiques et Minières.
Comme les constructions humaines, le massif rocheux peut être assimilé à un assemblage d'éléments, ceux-ci diversifiés éventuellement par leurs formes et leurs matériaux La description structurale nécessaire pour prévoir le comportement mécanique du massif comprend donc deux parties : description des matériaux, composites naturels, description des discontinuités. Par rapport aux composites artificiels, les roches sont moins diversifiées, en ce que les fibres n'y jouent qu'un rôle épisodique. De même, les assemblages naturels du massif rocheux sont moins diversifiés que les assemblages utilisés en construction, mais en revanche les caractères des roches et des massifs sont beaucoup plus dispersés et beaucoup plus difficiles à connaitre avec la précision souhaitable.

Les édifices naturels sont souvent étroitement liés à la structure du massif, par exemple les cavités karstiques suivant les diaclases principales. Le terrain alentour est donc massif et le toit est généralement un joint de stratification. La position et la forme des versants naturels dépendent aussi très largement de leur structure par exemple les parois verticales sont plus fréquentes lorsque la stratification est horizontale (exemple remarquable de Monument Valley aux confins du Colorado et du Nouveau Mexique).

Parmi les édifices artificiels, les cavités minières peuvent tirer avantage de certains éléments structuraux, dans la mesure où l'exploitation est précisément un démontage sélectif du massif rocheux Ainsi dans l'exploitation de couches horizontales, la stabilité des piliers n'est pas sensible en premier lieu à la simple présence de joints de stratification, tandis que la stabilité du toit dépend de l'épaisseur et de la nature de bancs parallèles à la cavité. Quelques autres ouvrages peuvent bénéficier aussi de structures favorables, par exemple les fondations sur couches horizontales, mais dans de nombreux cas la structure apparaît au contraire défavorable et/ou la liberté de choix du projeteur est trop limitée pour qu'une adaptation de l'ouvrage au site soit possible.

La stabilité des cavités est toutefois un cas assez général où les écarts à la continuité, l'isotropie et l'homogénéité paraissent les moins susceptibles d'effets dommageables. Des raisonnements simples le montrent pour l'effet des discontinuités et des anisotropies. Quant aux hétérogénéités, elles sont étudiées ci-dessous sous une forme schématisée, par la méthode des éléments finis. 


\section{Distribution des contraintes en terrain homogène}

On sait que le creusement d'une cavité dans un milieu élastique où règne un champ de contraintes ne modifie sensiblement l'état initial des contraintes qu'au voisinage des parois. II est utile de préciser quelque peu cette notion de voisinage puisque c'est le domaine dans lequel la présence de "défauts" peut jouer un rôle appréciable.

La distribution des contraintes est classique avec les hypothèses simplificatrices du problème plan isotrope: milieu continu, homogène, isotrope, élastique, infini, non pesant mais sous contrainte isotrope constante $p$, cavité circulaire de rayon $R$ (fig. 1 a-a').

Le problème est de révolution : sur tout rayon la contrainte radiale est nulle sur le contour et se rapproche de $p$ en $1 / r^{2}$; la contrainte tangentielle est $2 p$ sur le contour, elle est toujours symétrique de la contrainte radiale par rapport à p. Si la figure $1 \mathrm{a}-\mathrm{a}^{\prime}$ est bien connue, elle n'est pas partout dessinée avec précision : la pente de la tangente à l'origine est \pm 2 (en prenant comme unités $R$ et $p$ sur les axes $\mathrm{Ox}$ et $\mathrm{Oy}$ ) ce qui met en évidence la forte variation de la contrainte dès qu'on s'éloigne du contour.

Cette variation est divisée par 9 à $3 R$ du centre du cercle, donc à 1 diamètre du contour. Au-delà, la contrainte initiale n'est donc pas modifiée d'une façon sensible (même si les déplacements le sont, car ils intègrent les déformations jusqu'à l'infini, et diminuent donc en $1 / r$; ils ne sont divisés par 9 qu'à $9 R$ du centre, c'est-à-dire à 4 diamètres du contour).

Cette théorie s'applique assez exactement à l'équilibre d'un puits vertical à grande profondeur, mais elle s'applique encore avec une précision acceptable, comme les calculs modernes l'ont montré, à l'équilibre de tunnels non superficiels.

La figure 1 a-a' permet de se représenter le voisinage de la cavité comme un ensemble d'anneaux circulaires se partageant la déformation élastique induite par l'ouverture de la cavité.

Dans la pratique, c'est surtout l'hypothèse de la symétrie de révolution qui est inadéquate : ni la contrainte initiale ni le milieu ne sont isotropes. Le seul problème d'anisotropie de la contrainte est brièvement rappelé ci-dessous.

Le cas général est obtenu par superposition. Soit donc (fig. 1 b) une contrainte monoaxiale $\sigma_{y}$ parallèle à l'axe Oy. La contrainte tangentielle sur le contour n'est plus constante, mais varie sinusoïdalement autour de $\sigma_{\mathrm{y}}$ :

$$
\sigma_{\theta}=\sigma_{\mathrm{v}}(1+2 \cos 2 \theta)
$$

où l'angle $\theta$ est compté à partir de $O x$. La valeur moyenne $\sigma_{y}$ est atteinte sur les bissectrices: le maximum $3 \sigma_{y}$ sur l'axe $O x$; le minimum $-\sigma_{y}$ sur l'axe $O y$; la contrainte tangentielle s'annule pour $\theta= \pm 60^{\circ}$; en outre la valeur $2 \sigma_{y}$ est obtenue pour $\theta= \pm 30^{\circ}$.

Ainsi, la contrainte sur le contour est diminuée sur deux quartiers, et augmentée sur les deux autres. En particulier. le coefficient multiplicateur dépasse 2 sur deux secteurs de $60^{\circ}$ et atteint 3 sur l'axe Ox. Quant à la variation de contrainte le long de l'axe $\mathrm{Ox}$, elle est beaucoup plus rapide que dans le cas précédent: la tangente au départ est en effet -7 au lieu de -2 ; la loi est :

$$
\frac{\sigma_{\theta}}{\sigma_{y}}=1+\frac{R^{2}}{2 r^{2}}+\frac{3 R^{4}}{4 r} \text { au lieu de } \frac{\sigma_{\theta}}{\mathrm{P}}=1+\frac{R^{2}}{r^{2}}
$$

L'étude de la zone de traction a peu d'intérêt car elle se rétrécit très vite lorsqu'existe une composante $\sigma_{x}$ et elle disparaît dès que

$$
\sigma_{\mathrm{x}}=\frac{\sigma}{2} \mathrm{y}
$$

Le cas qui sera étudié ci-dessous comporte une composante $\sigma_{x}=\sigma_{y}$ La dissymétrie du cas monoaxial est alors atténuée. Sur le contour, la contrainte tangentielle est:

$$
\sigma_{\theta}=\sigma_{y}+\sigma_{x}+2\left(\sigma_{y}-\sigma_{x}\right) \cos 2 \theta=\sigma_{y}(1,5+\cos 2 \theta)
$$

Le maximum sur $O x$ est $2,5 \quad \sigma_{y}$; le minimum sur $O y$, $0,5 \sigma_{y}$; les valeurs remarquables $2 \sigma_{y}, 1,5 \sigma_{y}$ et $\sigma_{y}$ sont obtenues respectivement pour des angles $\theta=30^{\circ}, 45^{\circ}$, $60^{\circ}$. Ainsi le coefficient multiplicateur dépasse 2 sur les mêmes secteurs de $60^{\circ}$ que dans le cas monoaxial, mais il est moins élevé, il atteint seulement 2,5 (fig. 1-c). La variation de contrainte le long de $\mathrm{Ox}$ est intermédiaire entre les deux précédentes. Elle est donnée par:

$$
\frac{\sigma_{\theta}}{\sigma_{y}}=1+\frac{3 R^{2}}{4 r^{2}}+\frac{3 R^{4}}{4 r^{4}}
$$

La tangente au départ a pour valeur $-4,5$; les trois courbes ont en commun le point $r=\sqrt{3 \bar{R}}, \sigma_{\theta}=4 / 3 \quad \sigma_{y}$, où elles se croisent. Ce point est d'ailleurs commun à tous les cas de contrainte biaxiale. Aussi suffit-il pour tracer une autre courbe de la famille de connaître la contrainte sur le contour, $3 \sigma_{y}-\sigma_{x}$, ce qui donne un coefficient multiplicateur $3-\frac{\sigma_{x}}{\sigma_{y}}$ et de savoir en outre que la tangente au début de la courbe passe par le point $r=1,2 R, \sigma=1,6 \sigma_{\mathrm{y}}$.

Le détail des exemples ci-dessus montre bien qu'une augmentation sensible de la contrainte tangentielle règne seulement dans un domaine limité. Si dans ce domaine se trouve une discontinuité ou une inclusion beaucoup plus déformable que le reste du milieu, une grande part des déplacements se localisera sur ce "défaut » et l'augmentation de contrainte sera diminuée.

Lorsque le dépassement de la limite élastique conduit à un comportement parfaitement plastique (sans perte de cohésion) le seuil de plasticité limite la contrainte tangentielle sur le contour, puis elle augmente rapidement dans l'épaisseur de l'anneau plastique (fig. 1, courbe d). Dans ce cas, la zone où la contrainte initiale est augmentée est repoussée au-delà de l'anneau plastique.

En réalité, ce n'est pas l'excès de contrainte qui est nécessaire mais seulement la déformation. La figure 1 met en évidence un stockage d'énergie plastique autour de la cavité. Mais si cette énergie est dissipée par glissement, déformation plastique ou rupture localisée, l'équilibre peut être conservé.

\section{Rappel d'études antérieures}

La concentration des contraintes de compression au voisinage des angles des cavités est combattue depuis longtemps par les mineurs grâce à un artifice classique: le creusement de trous de mine parallèles très rapprochés suivant la bissectrice de l'angle. Cet artifice peut être schématisé comme un abaissement du module de déformation du matériau, c'est-à-dire la création d'une inclusion très déformable. D'ailleurs, le même artifice a été employé dans des modèles de mécanique des roches: grâce à des trous plus ou moins rapprochés, le module moyen du milieu peut être modifié à volonté. Dans ces modèles, le comportement élastique est seul utilisé, tandis que dans la mine, un comportement irréversible est acceptable allant jusqu'à l'écrasement des "piliers" ou cloisons entre les trous et la fermeture presque totale d'une sorte de saignée si les cloisons sont très minces. Seule compte en effet l'ampleur de la première déformation.

Le même artifice a été utilisé aussi dans des ouvrages non miniers, par exemple des usines hydroélectriques souterraines, la première fois à Poatina (Tasmanie), pour atténuer une concentration de contrainte le long du toit. II a été recommandé aussi (mais semble-t-il pas encore utilisé) pour diminuer la décompression autour des tunnels profonds (Dessenne et Duffaut, 1970).

Un premier calcul par éléments finis a porté sur linfluence de saignées radiales de longueur limitée (c'est-à-dire d'inclusions de module nul) (Aufaure et al, 1972). Dans le cas d'une contrainte isotrope autour d'un tunnel circulaire, le calcul a montré qu'il fallait au moins cinq saignées équidistantes pour que leur influence soit étendue à tout le périmètre. La zone d'influence d'une saignée est ainsi apparue de l'ordre de $70^{\circ}$. Le même calcul a montré que la longueur des saignées est importante. Les saignées de longueur R/5 sont plus efficaces que celles de longueur $R / 10$. L'ouverture des saignées était $R / 1000$. 

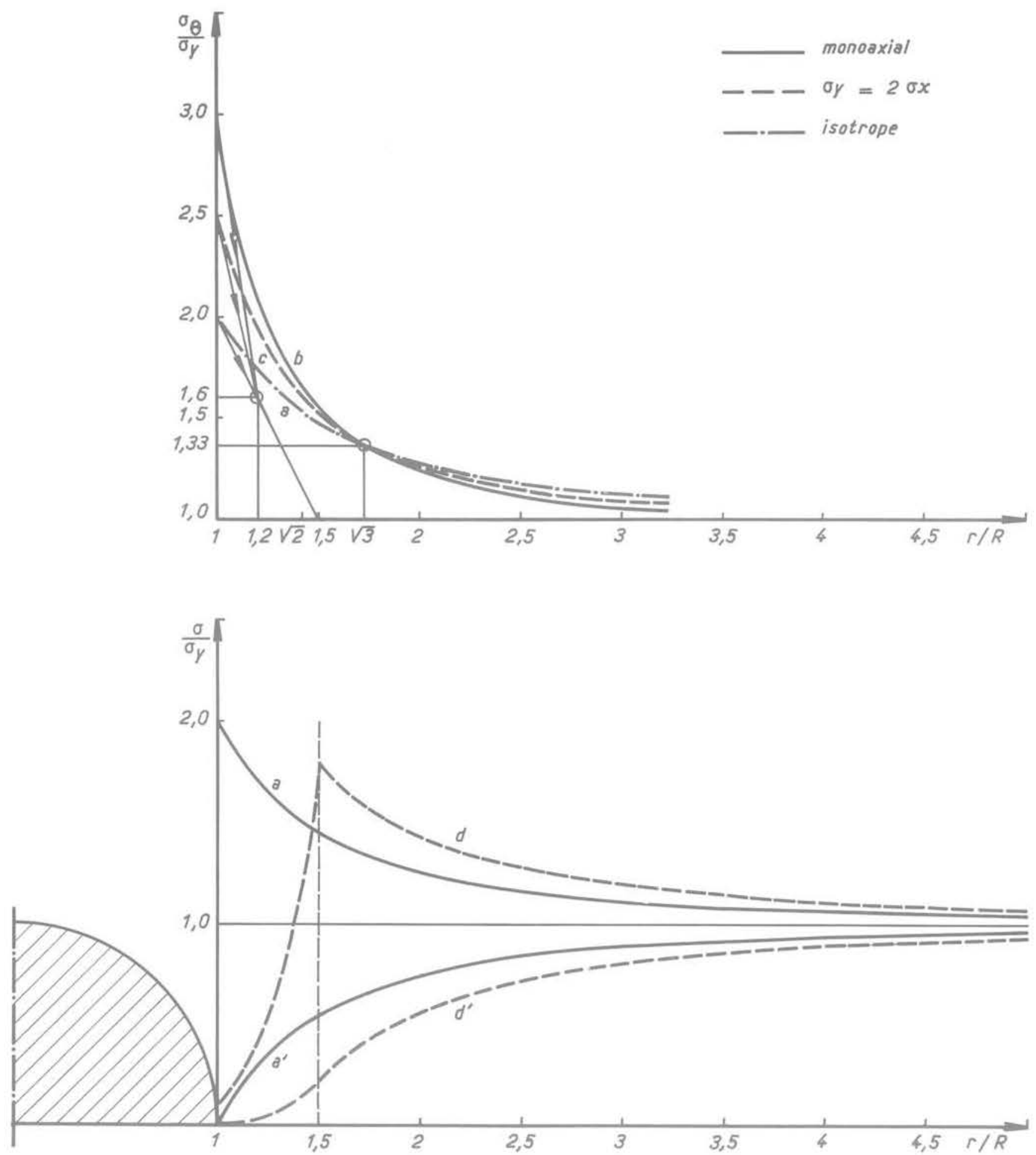

$a, a^{\prime}$ : Problème de révolution - contraintes tangentielle a et radiale $a^{\prime}$

b : Problème monoaxial - contrainte tangentielle

c : Problème biaxial $\sigma y=2 \sigma x$ - contrainte tangentielle

$d, d^{\prime}$ : Equilibre élostique - plastique de révolution, angle de frottement $50^{\circ}$, cohésion $0,1 \sigma_{\gamma}$, contraintes tangentielle ot radiale $d^{\prime}$.

Fig. 1 Distribution des contraintes au large d'une cavité circulaire 
Bien entendu, la symétrie de révolution est peu favorable à la mise en œuvre de saignées et la nature ne fournit jamais d'inclusions disposées à l'avance suivant cette symétrie, par contre un état de contrainte biaxial localise les concentrations de contraintes dans deux secteurs opposés. Une seule paire de saignées ou d'inclusions devrait donc se montrer efficace, ou par exemple une couche mince déformable placée suivant l'axe perpendiculaire à la contrainte principale majeure.

Un deuxième calcul par éléments finis a donc porté sur un problème orthotrope (deux axes de symétrie rectangulaires) (Dejean M. et Duffaut P., 1974). La contrainte étudiée était comme ci-dessous une contrainte biaxiale avec $\sigma_{y}=2 \sigma_{x}$. La paire de saignées était remplacée par une mince bande plus déformable de longueur infinie placée de part et d'autre de l'axe Ox. Le calcul a porté principalement sur les cas suivants:

- bande de module $E / 10$ ou $E / 100$

- bande d'épaisseur R/9 ou R/20

La figure 2 groupe les résultats, en ce qui concerne seulement la contrainte tangentielle le long du contour (qui est portée radialement à partir du contour, il ne s'agit donc pas d'une représentation polaire de $\sigma_{\theta}$ mais de $R+\sigma_{\theta}$ ). On constate sur cette figure que la concentration de contrainte est sensiblement diminuée par une bande de module $E / 10$, sur un secteur de $70^{\circ}$ environ, conformément aux premiers résultats sur les saignées. La largeur plus grande de la bande n'a guère d'effet supplémentaire qu'aux environs de $45^{\circ}$, son effet s'exerce donc sur un secteur un peu plus largement ouvert, proche de $90^{\circ}$.

Quant à la bande à module $E / 100$, son effet est très marqué, elle diminue à peu près de moitié la concentration de contrainte sur un secteur de $90^{\circ}$. En fait, son effet semblait se faire sentir sur tout le périmètre, au point que la compression sur l'axe Oy était proche de 0 . Ce résultat peu favorable n'a pas été retrouvé et pourrait s'expliquer par une modélisation moins précise.

Un joint sans frottement avait été introduit aussi à la limite de la bande, mais son effet est resté négligeable comme il est facile de le comprendre, car il était trop proche d'un axe de symétrie sur lequel les cisaillements sont nuls.

\section{Effet de la longueur d'inclusions symétriques}

A lá suite de ces études il a paru souhaitable d'élargir la portée de leurs résultats en précisant quelques aspects supplémentaires de l'effet d'inclusions très déformables, qu'il s'agisse d'inclusions naturelles ou artificielles. La troisième série de calculs a été conduite comme les précédentes par éléments finis et avec le même état de contrainte orthotrope $\sigma_{y}=2 \sigma_{x}$. Elle a porté sur un ensemble d'inclusions de module $E / 100$ et d'épais-seur $R / 20$,

a en fonction de la longueur de l'inclusion qui a pris les valeurs $R / 2, R, 2 R, 4 R$ et infinie pour une même position idéale sur l'axe $O x$;

b en fonction de l'angle de l'inclusion lorsqu'elle s'écarte de la position idéale : cet angle a pris les valeurs $30^{\circ}, 45^{\circ}$ et $60^{\circ}$ sur $0 x$.

La première comparaison à laquelle a été ajoutée une inclusion de module nul (saignée) de longueur $R / 2$, a été traitée symétriquement par rapport à Oy, ce qui a permis de borner le modèle à un seul quadrant puisque la figure est aussi symétrique par rapport à Ox. Par contre, la deuxième comparaison a considéré seulement des inclusions de longueur infinie mais d'un seul côté du cercle, aussi le modèle a-t-il comporté les 4 quadrants.
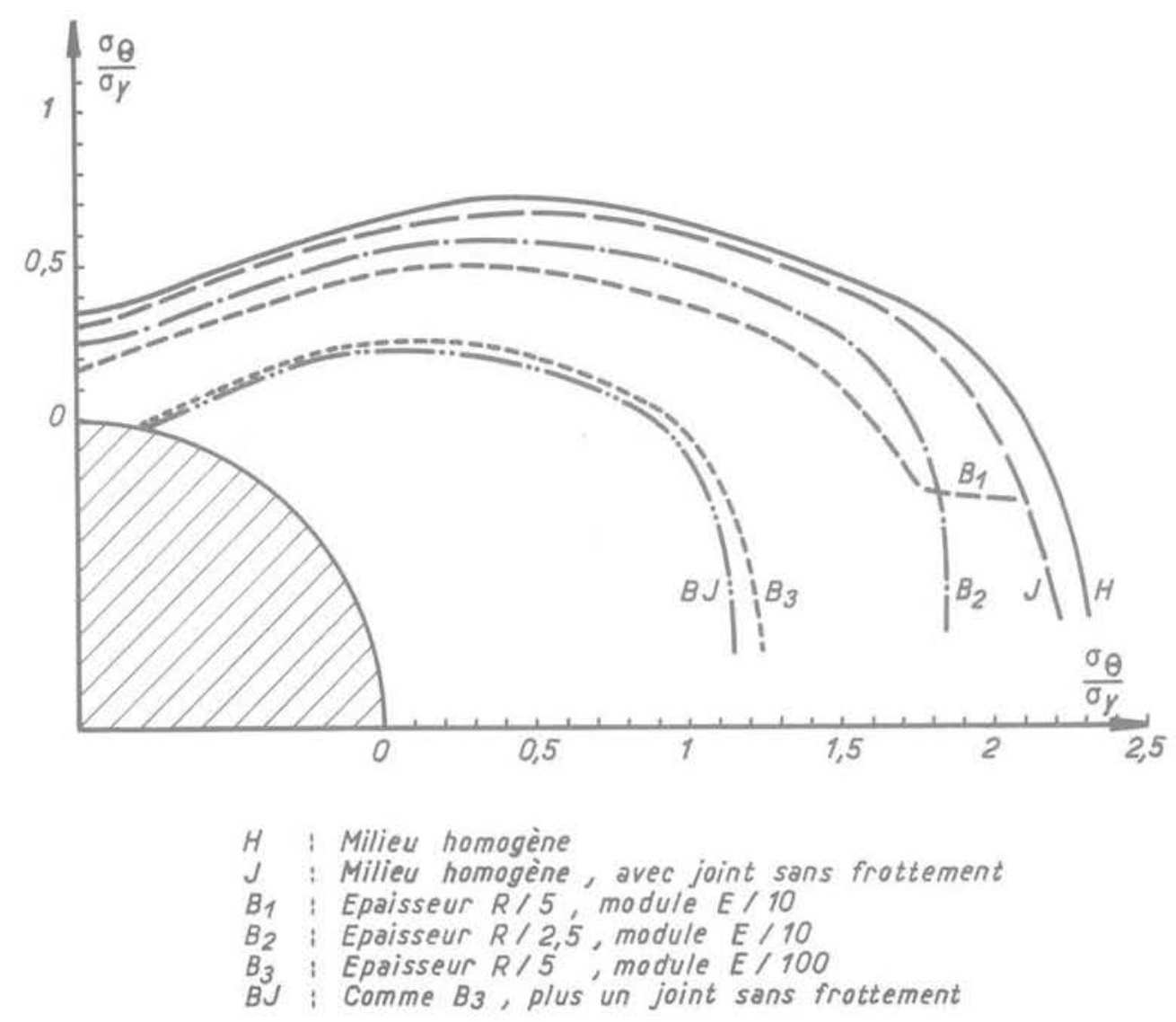

Fig. 2 Contraintes tangentielles le long du pourtour d'une galerie circulaire Problème biaxial, $\sigma_{x}=\sigma_{y} / 2$ toutes les inclusions sont planes et symétriques 
La figure 3 représente le modèle utilisé. Ses limites, sur lesquelles les déplacements sont nuls ont été prises à 13 diamètres du centre. Le contour du cercle est remplacé par un polygone à 84 côtés. Le nombre de nœuds est 399 pour le quart de modèle, et 546 pour le modèle entier.

La distribution de contraintes dans un milieu homogène permet de vérifier la validité du modèle : les contraintes de compression sur le contour varient entre un maximum de 2,3 au lieu de 2,5 sur l'axe Ox et un minimum de 0,54 au lieu de 0,5 sur l'axe Oy (valeurs exprimées relativement à $\left.\sigma_{y}\right)$. Ces petites différences traduisent l'approximation qui est faite sur la forme de la courbe et les limites du modèle. En fait, le lissage des courbes atténue ces différences, les résultats étant alternativement par excès ou par défaut.

Les résultats exploités ci-dessous concernent exclusivement les contraintes tangentielles sur le contour puisque c'est là que la concentration de contrainte $\sigma_{\theta} / \sigma_{\mathrm{y}}$ est la plus forte. Les listings permettent évidemment de détailler les résultats dans toute l'étendue du modèle. La figure 4 utilise la même représentation que la figure 2 : sur chaque rayon $\sigma_{\theta} / \sigma_{\mathrm{y}}$ est porté à partir du contour.

Cette figure montre que l'effet des inclusions horizontales est nettement différencié suivant les secteurs du contour :

- dans le secteur de $60^{\circ}$ autour de $\mathrm{Ox}$, où la concentration de contrainte initiale dépasse 2 , celle-ci est divisée par deux par les inclusions, quelle que soit leur longueur. Si en effet les courbes se rangent bien dans l'ordre des longueurs, l'écart relatif est faible. On peut en conclure qu'une inclusion de longueur inférieure à $\mathrm{R} / 2$ aurait encore une influence appréciable.

- dans le secteur de $60^{\circ}$ autour de Oy, l'effet des inclusions courtes est peu sensible mais par contre la longueur de l'inclusion ne joue plus un rôle négligeable. L'inclusion infinie abaisse de près de moitié la compression sur Oy. Un résultat plus net encore mais douteux avait été obtenu précédemment,

- enfin dans le secteur de $60^{\circ}$ autour de la bissectrice intermédiaire entre les deux précédents, la seule courbe correspondant à la longueur $\mathrm{R} / 2$ est nettement séparée des autres. Dans ce domaine, une inclusion de longueur au moins égale à $R$ est donc nettement plus efficace.

La même figure porte aussi le cas d'une saignée de longueur $R / 2$, dont l'influence apparaît bien supérieure à celles de toutes les inclusions étudiées.

\section{Effet d'une inclusion dissymétrique, inclinée ou non}

La figure 5 présente les résultats de la même façon que les figures 2 et 4 . Elle porte comme même référence la courbe du milieu homogène, seule symétrique par rapport aux deux axes (1).

Quelle que soit l'inclinaison de l'inclusion, on constate d'abord une diminution de la concentration de contrainte du côté de l'inclusion, et une augmentation corrélative de l'autre côté. Cet effet est le seul sensible sur tout le secteur gauche, il est maximal pour une inclusion horizontale et ne dépasse pas $20 \%$ en moins ou en plus sur le maximum de la concentration de contrainte. A cela près, l'effet de l'inclusion horizontale est presque identique à celui étudié au chapitre précédent.

Les inclusions inclinées ont un effet marqué dans le secteur autour de la bissectrice et un moindre effet dans les secteurs autour de Ox et de Oy. II est remarquable que ces effets autour de $\mathrm{Ox}$ et de Oy soient pratiquement les mêmes au-dessus et au-dessous de Ox. Cet effet d̀ distance diminue lorsque l'angle sur $\mathrm{Ox}$ augmente. En réalité, ces inclusions inclinées, et surtout celle placée

1) Cette courbe est toutefois perturbée dans la zone où les inclusions ont été placées car le maillage initial a été distordu pour pouvoir les modéliser. à $45^{\circ}$, sont soumises principalement à une déformation de cisaillement. Aussi trouve-t-on systématiquement une grande variation de la contrainte tangentielle à la traversée de l'inclusion.

La même figure porte l'effet d'une saignée de longueur $R / 2$ d'un seul côté ; comme au chapitre précédent, cet effet est de beaucoup supérieur à celui des inclusions de module non nul.

\section{Interprétation pratique}

Lorsque la concentration de contrainte à la paroi d'une cavité dépasse la résistance à la compression monoaxiale du matériau, ce matériau se brise s'il est fragile, il cède et se déforme s'il est parfaitement plastique. La rupture fragile a été décrite dans les roches continues comme certains massifs de granite (tunnel routier du Mont Blanc) ou même la craie, et dans ce cas avec un recouvrement beaucoup plus faible, $100 \mathrm{~m}$ au lieu de 2000 . Les grandes déformations ont été décrites surtout dans les roches schisteuses (tunnel de chemin de fer du Simplon, tunnel routier des Tauern) et dans les roches argileuses à profondeur beaucoup plus faible, $20 \mathrm{~m}$ au lieu de 500 par exemple. Mais en réalité un très grand nombre de tunnels apparaissent stables, bien qu'ils soient creusés à une profondeur où la résistance à la compression du terrain devrait être dépassée à la paroi. Cette observation s'explique par des ruptures et/ou des " grandes déformations " qui restent localisées et passent inaperçues, faute d'un système de mesure adéquat, mais qui suffisent pour éliminer l'excès de contrainte dans la zone limitée où il aurait dû exister.

Beaucoup de terrains en effet présentent des structures qui ressemblent aux schémas étudiés ci-dessus, et qui ont pratiquement les mêmes effets (fig. 6):

La bande horizontale étudiée peut être déplacée par rapport à l'axe de symétrie sans perdre son efficacité (6a). Elle peut être multiple, l'épaisseur cumulée de bandes plus mince étant équivalente à celle de la bande unique (6b). Elle peut être interrompue, sous forme de tirets (6c).

L'inclinaison de la bande est efficace comme on l'a vu dans de larges limites (6d). En particulier entre 30 et $60^{\circ}$ sur les axes de symétrie, même un joint peut jouer un rôle efficace $(6 e)$

Ce sont seulement les bandes parallèles ou subparallèles à la contrainte majeure qui sont sans effet (car elles ne sont pas déformées par cette contrainte) et peut être les bandes dont la direction est favorable mais qui coupent le contour dans les zones les moins chargées.

Dans tous ces cas de figures, les défauts du contour acceptent l'essentiel du raccourcissement et évitent qu'un effort excessif soit supporté par le matériau principal. $\mathrm{Ce}$ comportement est très exactement celui des matériaux d'emballage. Placés en série avec le matériau à protéger, les matériaux d'emballage acceptent une grande déformation au cours de laquelle l'effort sur le matériau fragile est limité.

Le même raisonnement s'applique au revêtement des tunnels. Dans les tunnels profonds, il a fallu parfois placer entre les voussoirs de béton des planchettes de bois susceptibles de s'écraser. Les saignées laissées dans le béton projeté au tunnel du Tauern ont le même objectif et sont tout-à-fait comparables aux saignées creusées dans le terrain lui-même. La mise en place entre le terrain et le revêtement d'un anneau susceptible de diminuer de volume, tel que l'a recommandé Muir Wood A. (1974) est une autre solution au même problème.

Une autre conséquence des études ci-dessus c'est que les calculs traditionnels des cavités sont toujours pessimistes: la pointe de compression qui est censée régner sur la paroi même de la cavité n'a guère de chances d'être réellement atteinte, hormis dans de rares cas particuliers, parmi lesquels les roches dépourvues à la fois de porosité et de fissures.

Ce phénomène est très général, les contraintes infinies que prévoit la théorie de l'élasticité aux points anguleux sont limitées automatiquement par les propriétés d'adaptation plastique ou pseudo-plastique des matériaux réels. 


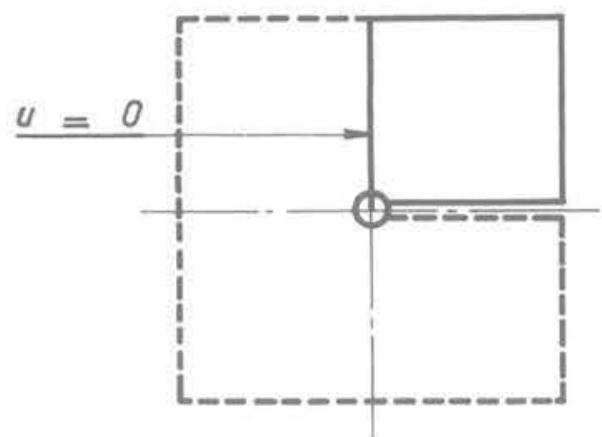

- Modèle entier -

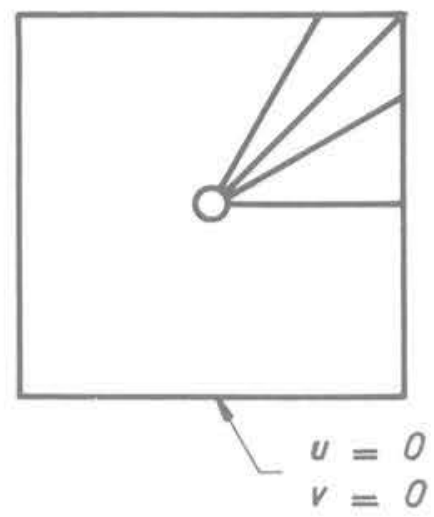

b _ INCLUSIONS DISSYMETRIQUES MODELE ENTIER

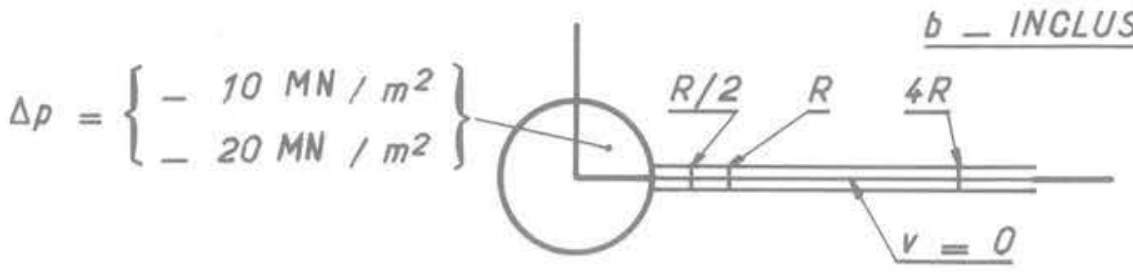

- Détail de l'inclusion -

- _ INCLUSIONS SYMETRIQUES - MODELE ORTHOTROPE

\section{LIMITE A UN QUARTIER}

Fig. 3 Modélisation pour le calcul par éléments finis

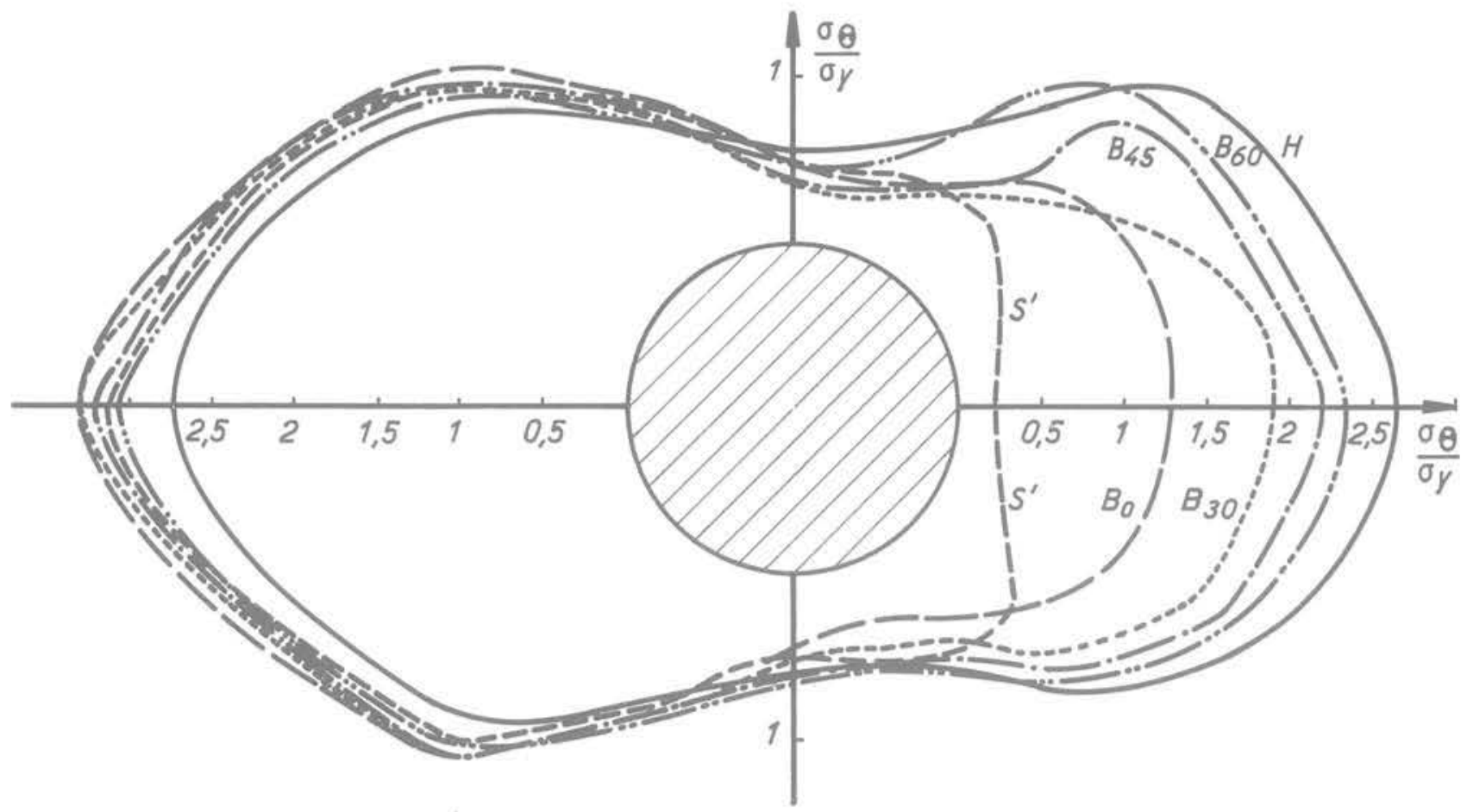

$H$ : Milieu homogène

B 60: Inclusion inclinée ¿ $60^{\circ}$

$B 45$ : Inclusion inclinée à $45^{\circ}$

B 30 inclusion inclinée d̀ $30^{\circ}$

BO, Inclusion de longueur infinie d'un seul côté

$S$ ' Saignée dissymétrique de longueur $R / 2$

Fig. 5 Contraintes tangentielles le long du contour d'une cavité circulaire Inclusions dissymétriques de longueur infinie 


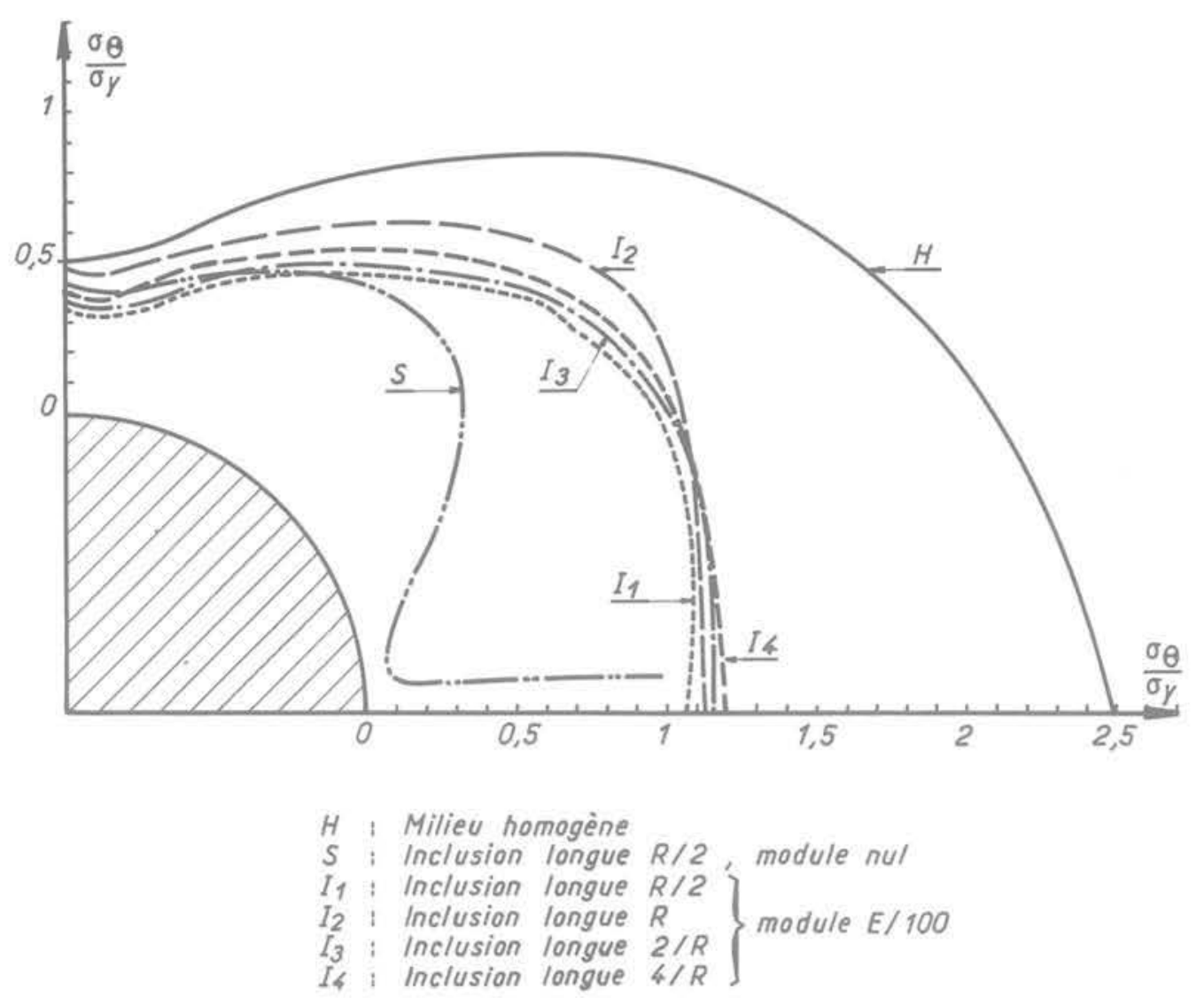

Fig. 4 Contraintes tangentielles le long du pourtour d'une galerie circulaire Problème biaxial, $\sigma_{x}=\sigma_{y} / 2$ toutes les inclusions sont planes et symétriques

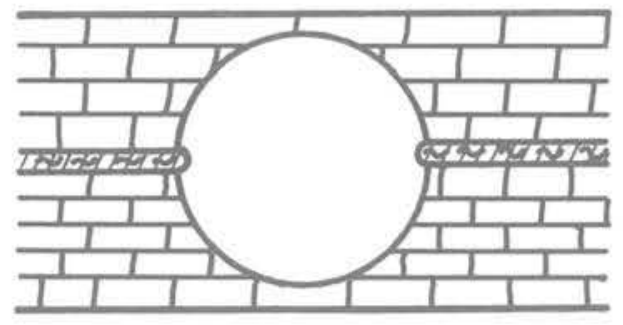

$-a$
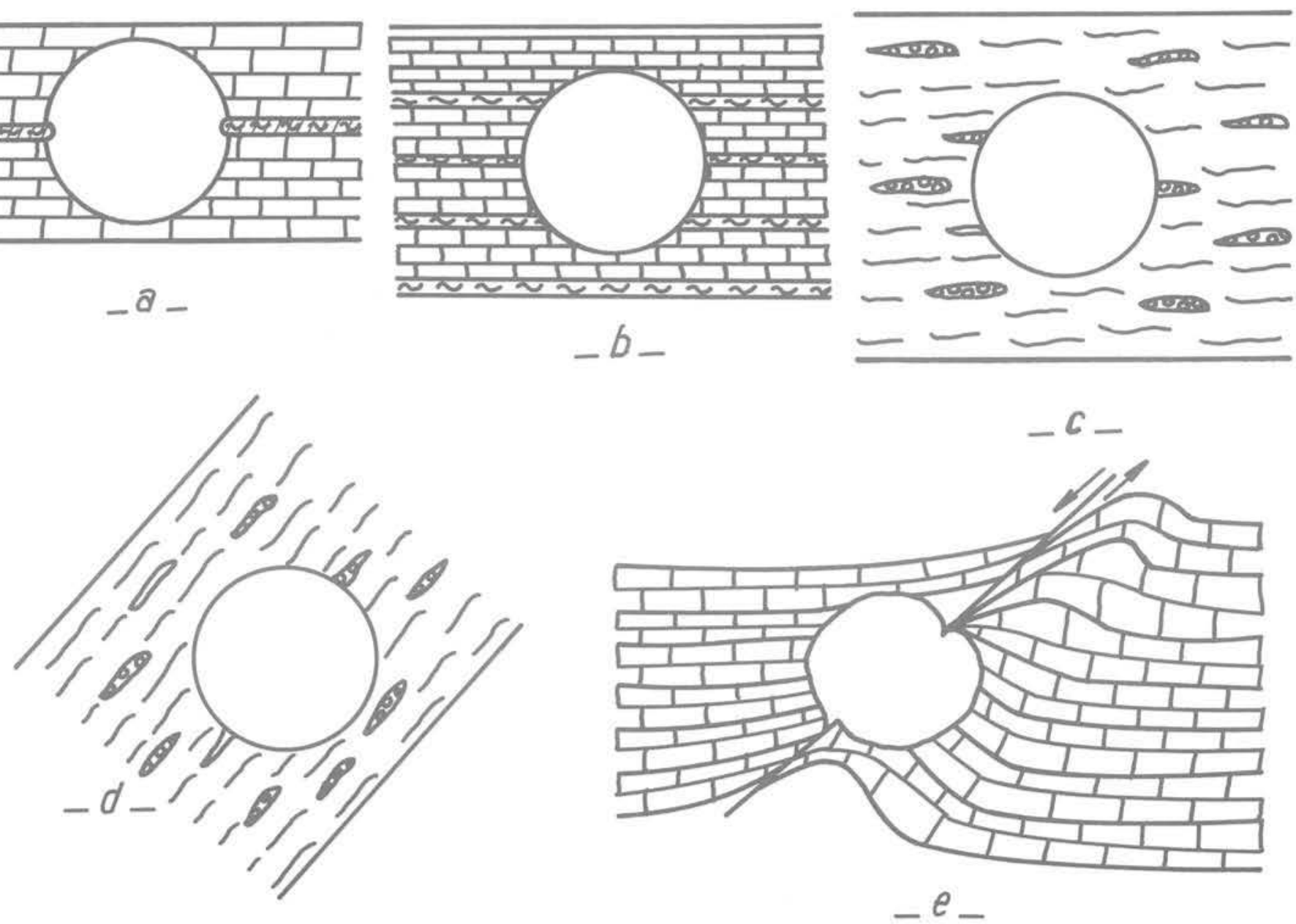

Fig. 6 Structures géologiques favorables à la stabilité des tunnels 
La stabilité des cavités profondes isolées est un problème local, limité aux environs immédiats de la cavité. Ce problème peut être résolu par le raccourcissement du contour sans qu'un soutènement ait un effort à exercer. II convient toutefois que le raccourcissement intervienne de préférence dans les zones où la concentration de contrainte apportée par la cavité est la plus grande.

Or. tout "défaut " du massif rocheux, c'est-à-dire toute discontinuité et toute inclusion susceptible de grande déformation, fut-ce un écrasement localisé, prend une part prépondérante à ce raccourcissement, et décharge d'autant le reste du massif.

Divers procédés ont été utilisés pour limiter les contraintes subies par les revêtements, dont plusieurs font appel à un matériau susceptible de s'écraser, exactement comme dans l'emballage des objets fragiles. Or. il convient de reconnaître que certaines structures géologiques comportent déjà un tel matériau d'emballage, à l'intérieur.

\section{Rèférences Bibliographiques}

AUFAURE M., BOZETTO J, and DUFFAUT P., Influence de saignées sur la répartition des contraintes autour d'une galerie, Symp. int. de la construction de cavités souterraines, Lucerne. 1972.

DEJEAN M. and DUFFAUT P., Valeurs extrêmes et stabilités des cavités. Journées géotechniques d'Orlèans, BRGM, déc. 1975.

DESSENNE J.-L. and DUFFAUT P., Les propriétés rhéologiques de la craie et leur influence sur le percement des galeries, La Houille Blanche, $n^{\circ} 5,1970$.

ENDERSBEE L.-A. and HOFTO E.-O., Civil engineering design and studies in rock mechanics for Poatina underground power station, Tasmania, Austral. Inst Engr. Conf., May 1963.

MUIR WOOD A., The circular tunnel in elastic ground, 1975 , Geotechnique 25, $n^{\circ} 1,115-127$

RABCEWICZ L. von, Diskussionsbeitrag, Symp. intern. de la construction des cavités souterraines, Lucerne, 1972. 